\title{
Primary frequency regulation with load-side participation Part II: beyond passivity approaches
}

\author{
Eoin Devane, Andreas Kasis, Marina Antoniou, and Ioannis Lestas
}

\begin{abstract}
We consider the problem of distributed generation and demand control for primary frequency regulation in power networks, such that stability and optimality of the power allocation can be guaranteed. It was shown in [1] that by imposing an input strict passivity condition on the net supply dynamics at each bus, combined with a decentralized condition on their steady state behaviour, convergence to optimality can be guaranteed for broad classes of generation and demand control dynamics in a general network.

In this paper we show that by taking into account additional local information, the input strict passivity condition can be relaxed to less restrictive decentralized conditions. These conditions extend the classes of generation and load dynamics for which convergence to optimality can be guaranteed beyond the class of passive systems, thus allowing to reduce the conservatism in the analysis and feedback design.
\end{abstract} trol.

Index Terms-frequency control, stability, decentralized con-

\section{INTRODUCTION}

In [1], we considered the problem of distributed generation and demand control in primary frequency regulation, focusing on mechanisms for which stability and optimality of the equilibrium points reached can be guaranteed. In particular, it was shown that by imposing an input strict passivity condition on the net supply dynamics at each bus, combined with a decentralized steady state condition on the input/output properties of these dynamics, convergence to the solution of an appropriately constructed network optimization problem can be guaranteed. A discussion of the motivation for considering this problem and existing literature on the topic can be found in [1, Section I]. To prove the convergence result in that work, we made use of Lyapunov techniques applied to the nonlinear system model, whereby the conditions imposed on the dynamics and the underlying system structure were exploited to construct a Lyapunov function for the network.

Passivity approaches often constitute a natural and useful framework through which one can investigate stability properties in power systems. The significance of such approaches has been recognized from an early stage as in e.g. [2], [3]. More recently, passivity studies in power systems have typically followed the framework of port-Hamiltonian systems, described in [4]. Examples of this approach include [5], [6], [7], [8]. Two additional interesting analyses, which illustrated

The research carried out was partly funded by an ERC starting grant.

Eoin Devane is with the Cambridge Centre for Analysis, Centre for Mathematical Sciences, University of Cambridge, Wilberforce Road, Cambridge CB3 0WA, United Kingdom; e-mail: esmd2@cam.ac.uk, Andreas Kasis is with the Department of Engineering, University of Cambridge, Trumpington Street, Cambridge, CB2 1PZ, United Kingdom; e-mail: ak647@cam.ac.uk, Marina Antoniou is with the Department of Engineering, University of Cambridge, Trumpington Street, Cambridge, CB2 1PZ, United Kingdom; e-mail: ma308@cam.ac.uk, Ioannis Lestas is with the Department of Engineering, University of Cambridge, Trumpington Street, Cambridge, CB2 1PZ, United Kingdom; e-mail: icl20@cam.ac.uk the application of passivity-based approaches to frequency control, can be found in [9], [10]. The former work generalized classical generation droop control to permit arbitrary passive controllers, while the latter used passivity arguments to demonstrate stability of a class of droop controllers in grid models where frequency regulation resources are shared amongst AC subgrids through HVDC links.

However, passivity assumptions can sometimes prove restrictive in power systems analysis, particularly when enforced on higher order models that are not necessarily passive. For this reason, within this paper we will investigate the question of what can be deduced without imposing such assumptions. As previously shown in [1], appropriate decentralized conditions on the steady state behaviour of the subsystems ensure that the equilibrium points are solutions of a corresponding network optimization problem. Additional conditions on the dynamic behaviour of the subsystems, such as passivity, are needed to ensure that these equilibrium points are also stable. Here, we will consider in more depth the latter problem: under what conditions are the equilibrium points asymptotically stable. Our approach within this paper, in contrast with that which we followed in [1], focuses on a linearization of the overall system about equilibrium and then makes use of frequency domain techniques to deduce stability. As such, we obtain conditions on the frequency response of transfer functions representing the generator and controllable load control dynamics under which convergence to optimality is guaranteed. Significantly, these conditions will be seen to be decentralized. If furthermore the network structure is known a priori, then we will see that even less conservative stability conditions can be derived. We will show that the conditions obtained can be seen, in an appropriate sense, as generalizations of the passivity conditions in [1], when a linearization is feasible, following from the additional local information taken into account in the analysis. This leads to decentralized rules that allow broader classes of generation and load control dynamics, thus reducing the conservatism in the analysis and design. It should be noted that the fact that the conditions derived are decentralized is a distinctive feature relative to classical approaches for small signal analysis in power systems [11]. This renders the problem much more involved and requires appropriate analysis tools to be developed.

The paper is organized as follows. The notation that will be used and various definitions are given in Section II. In Section III, we introduce the power network model and the classes of dynamics that will be considered and we recall the optimization interpretation of the system equilibrium points. In Section IV, we interpret the network dynamics as a feedback interconnection of appropriate subsystems, and we use this to obtain our main stability results. The proofs are given in the appendix. A discussion of the connections with the passivity analysis in [1], along with a numerical example are given in 
Section V. Finally, we draw conclusions in Section VI.

\section{NOTATION AND DEFINITIONS}

Within the paper we use $\mathbb{R}$ to denote the real numbers and $\mathbb{C}$ for the complex numbers. The set of non-negative real numbers is denoted by $\mathbb{R}_{+}$, and its closure $\overline{\mathbb{R}}_{+}$denotes the set $\mathbb{R}_{+} \cup\{+\infty\}$. The real and imaginary part of a complex number $z$ are denoted by $\Re(z)$ and $\Im(z)$ respectively. The imaginary unit is denoted by $j$. We use $\mathbb{R}^{n}$ and $\mathbb{C}^{n}$ to denote the sets of $n$-dimensional vectors with real and complex entries respectively. The notation $\left(q_{i}\right)_{i \in \mathcal{I}}$ denotes the vector with elements $q_{i}$ for $i$ in the index set $\mathcal{I}$. We also use $[x]_{a}^{b}$ to denote the projection of $x \in \mathbb{R}$ to $[a, b]$, i.e. $[x]_{a}^{b}:=\max \{\min \{x, b\}, a\}$.

For a function $f(q)$, the expression $f^{-1}(w)$ represents the preimage of the point $w$ under the function $f$, i.e. $f^{-1}(w)=$ $\{q: f(q)=w\}$. When the function $f$ is invertible, $f^{-1}$ then defines the inverse function of $f$. The first derivative of a differentiable function will be written $f^{\prime}(q)=\frac{d}{d q} f(q)$.

For a time-varying quantity $q(t)$, we write $\dot{q}$ for the derivative $\frac{d}{d t} q(t)$. For the stability analysis in Section IV, we work in the Laplace domain. The Laplace transform of $q(t)$ is written as $\hat{q}$. Transfer functions relating the Laplace transforms of time-varying quantities will be written as functions of the variable $s$. A superscript ${ }^{T}$ indicates the transpose of a matrix, and $\operatorname{diag}(y)$ for $y \in \mathbb{C}^{n}$ represents the diagonal matrix with diagonal entries $y_{i}$. Also for convenience in the presentation we denote $1 / y$ the vector with $i^{\prime}$ th element $1 / y_{i}$. We will require a number of notions from matrix analysis [12]. Firstly, we write $\sigma(Q)$ for the spectrum of a square matrix $Q$, i.e. the set of all eigenvalues of $Q$. If $\sigma(Q) \subseteq\{z \in \mathbb{C}: \Re(z) \geq 0\}$, then we say $Q$ is positive semidefinite and write $Q \succeq 0$. The spectral radius of $\mathbf{Q}, \rho(Q)$, is the maximum absolute value of the elements in $\sigma(Q)$. Also $\|Q\|_{\infty}$ denotes the induced infinity norm, which is equal to the maximum row sum $\max _{i} \sum_{j}\left|Q_{i j}\right|$, and we note that $\rho(Q) \leq\|Q\|_{\infty}$. The numerical range of $Q$ is the set $\left\{\frac{y^{\dagger} Q y}{y^{\dagger} y}: y \in \mathbb{C}^{n} \backslash\{0\}\right\}$, where $y^{\dagger}$ is the conjugate transpose of $y$. Finally, the convex hull of a set $S$, denoted by $\operatorname{Co}(S)$, is the smallest convex set that contains $S$.

Due to the fact that the system models we consider can, in general, have a continuum of equilibrium points, the precise concept of stability that will be of interest is as stated in the following definition. Within the definition, the distance of a point $q$ from a set $S$ is $\|q-S\|:=\inf _{s \in S}\|q-s\|$ for any convenient norm $\|\cdot\|$.

Definition 1: A set $S$ is said to be asymptotically stable for a differential equation system $\dot{q}=F(q)$ if:

i) For all $\epsilon>0$, there exists $\delta>0$ such that whenever $\|q(0)-S\|<\delta,\|q(t)-S\|<\epsilon$ for all $t \geq 0$,

ii) There exists $\eta>0$ such that whenever $\|q(0)-S\|<\eta$, $\lim _{t \rightarrow \infty}\|q(t)-S\|=0$,

both hold for all solutions $q(t)$ of the differential equation.

\section{PROBLEM FORMULATION}

\section{A. System model}

We consider a power network model described by a graph $(N, E)$, where $\mathcal{N}=\{1,2, \ldots,|N|\}$ is the set of buses and $\mathcal{E} \subseteq \mathcal{N} \times \mathcal{N}$ the set of transmission lines connecting the buses. The set $\mathcal{N}$ consists of generation buses $\mathcal{G}=\{1,2, \ldots,|\mathcal{G}|\}$ and load buses $\mathcal{L}=\{|\mathcal{G}|+1, \ldots,|\mathcal{N}|\}$. Furthermore, we use $(i, j)$ to denote the link connecting buses $i$ and $j$ and assume that the graph $(\mathcal{N}, \mathcal{E})$ is directed with arbitrary direction, so that if $(i, l) \in \mathcal{E}$ then $(l, i) \notin \mathcal{E}$. For each $i \in \mathcal{N}$, we use $l: l \rightarrow i$ and $l: i \rightarrow l$ to denote the sets of buses that are predecessors and successors of bus $i$ respectively. The form of the dynamics introduced in (1)-(2) below is unaltered by any change in the graph ordering, and all of our results are independent of the choice of direction. We also assume that $(\mathcal{N}, \mathcal{E})$ is connected, that bus voltage magnitudes are $\left|V_{i}\right|=$ 1 p.u. for all $i \in \mathcal{N}$, that all lines $(i, l) \in \mathcal{E}$ are lossless and characterized by their susceptances $B_{i l}=B_{l i}>0$, and that reactive power flows do not affect bus voltage phase angles and frequencies.

We then recall the formulation of the power system model considered in [1]. This consists of the swing equation dynamics (e.g. [13])

$$
\begin{gathered}
\dot{\eta}_{i l}=\omega_{i}-\omega_{l},(i, l) \in \mathcal{E}, \\
M_{i} \dot{\omega}_{i}=-p_{i}^{L}+s_{i}-\sum_{l: i \rightarrow l} p_{i l}+\sum_{l: l \rightarrow i} p_{l i}, i \in \mathcal{N}, \\
p_{i l}=B_{i l} \sin \eta_{i l}-p_{i l}^{n o m},(i, l) \in \mathcal{E},
\end{gathered}
$$

coupled with a general class of dynamics with inputs given by the negative local frequency measurements $-\omega_{i}$,

$$
\begin{aligned}
& \dot{x}^{i}=f^{i}\left(x^{i},-\omega_{i}\right), \\
& s_{i}=g^{i}\left(x^{i},-\omega_{i}\right) .
\end{aligned}
$$

Within the system (1)-(2), the quantity $p_{i}^{L}$ represents a step change in frequency-independent, uncontrollable demand in the system. The variables $\eta_{i l}, \omega_{i}$, and $p_{i l}$ are time-dependent quantities representing, respectively, the power angle difference between buses $i$ and $l$, the deviation from a constant nominal value ${ }^{1} \omega_{i}^{\text {nom }}$ of the system frequency at bus $i$, and the deviation from a nominal value $p_{i l}^{n o m}$ of the power transfer from bus $i$ to bus $l$. The constant $M_{i}$ denotes generation inertia, and hence is zero for all $i \in \mathcal{L}$ and strictly positive for all $i \in \mathcal{G}$.

The outputs in (2), $s_{i}$, represent deviations from nominal values of the net power supply to the system at the bus $i \in \mathcal{N}$. As discussed explicitly in [1], these supply variables comprise contributions from power generation, controllable demand, and frequency-dependent, uncontrollable demand. Since our purpose here is to investigate the stability properties of the system (1)-(2), we suppress these terms and, without loss of generality, work just with the combined supply variables $s_{i}$ directly for simplicity of the presentation. Analogously, the subsystem (2) includes the aggregate dynamics associated with the supply variables mentioned above. Here, the vector quantities $x^{i}$ represent internal states, and the functions $f^{i}$ and $g^{i}$ are assumed to be locally Lipschitz and continuous respectively.

We assume that each of the subsystems in (2) has the property that given any constant input, the subsystem has a unique locally asymptotically stable equilibrium point. The

\footnotetext{
${ }^{1}$ These nominal values correspond to an equilibrium point of (1)-(2) with a constant, uniform frequency that represents the target frequency at which we wish the system to operate. In Europe this would typically be $50 \mathrm{~Hz}$, while in North America it is $60 \mathrm{~Hz}$.
} 
uniqueness assumption is made here for simplicity and can be relaxed to a condition that these equilibria are isolated. For each constant frequency input $-\bar{\omega}_{i}$, we denote the corresponding equilibrium state vector $x^{i}$ by the static input-state characteristic map $\kappa^{i}\left(-\bar{\omega}_{i}\right)$. That is, $f^{i}\left(\kappa^{i}\left(-\bar{\omega}_{i}\right),-\bar{\omega}_{i}\right)=0$. In terms of these, we can also define the static input-output characteristic maps

$$
k^{i}\left(-\bar{\omega}_{i}\right):=g^{i}\left(\kappa^{i}\left(-\bar{\omega}_{i}\right),-\bar{\omega}_{i}\right) .
$$

That is, the input-output characteristics (3) represent the unique steady state responses obtained in (2) under the constant inputs $-\bar{\omega}_{i}$.

\section{B. Equilibrium points}

Within the paper we are interested in the stability properties of equilibrium points of system (1)-(2). In the remainder of the paper, the obvious vectorized notation is used, where subscripts are omitted to denote the corresponding vectors.

Definition 2: An equilibrium of the system (1)-(2) is a collection of constants $\left(\eta^{*}, \omega^{*}, x^{*}\right)$ at which the right-hand sides in (1)-(2) are all equal to zero.

Henceforth, we assume that there exists some equilibrium, and write $p^{*}$ and $s^{*}$ for the values at this equilibrium of the corresponding quantities defined in (1c) and (2). We then suppose that the equilibrium considered has power flows satisfying a security constraint.

Assumption 1: $\left|\eta_{i l}^{*}\right|<\frac{\pi}{2}$ for all $(i, l) \in \mathcal{E}$.

In the case $i \in \mathcal{L}$, the generation inertia $M_{i}$ is zero and so (1b) reduces to an algebraic condition. Therefore, in order to ensure that the system (1)-(2) has a well-defined state space realization, we impose another assumption constraining this algebraic condition to have locally unique solutions. In the following assumption $\omega^{G}$ and $\omega^{L}$ denote the vectors of frequencies at the load and generator buses respectively.

Assumption 2: There exists an open neighbourhood $R$ of $\left(\eta^{*}, \omega^{G, *}, x^{*}\right)$ such that at any time instant $t, \omega^{L}(t)$ is uniquely determined by the system states $\left(\eta(t), \omega^{G}(t), x(t)\right) \in T$ and equations (1)-(2), and the map relating the system states to $\omega^{L}(t)$ is locally Lipschitz.

Remark 1: It was discussed in [1] that Assumption 2 is a condition that can often be easily checked by algebraic means. It is required in the analysis that follows to ensure that the transfer functions at the load buses do not become degenerate.

\section{Optimality interpretation}

One of our main results in [1] was to show that the equilibria defined in Definition 2 are optimal for an appropriately constructed economic minimization problem. In the simplified notation introduced above, this problem can be formulated as

$$
\underline{\text { OSLC: }}\left\{\begin{array}{l}
\min _{s} \sum_{i \in \mathcal{G}} c_{i}\left(s_{i}\right) \\
\text { subject to } \sum_{i \in G} s_{i}=\sum_{i \in N} p_{i}^{L}, \\
s_{i}^{\text {min }} \leq s_{i} \leq s_{i}^{\text {max }}, \forall i \in \mathcal{N},
\end{array}\right.
$$

where $c_{i}\left(s_{i}\right)$ is a function representative of the cost incurred for the net supply deviation $s_{i}$ at bus $i \in \mathcal{N}$. The full detail of how this cost is split between the controllable generation and load control terms and the uncontrollable frequency-dependent loads was discussed in [1].

If each cost $c_{i}$ is assumed to be a continuously differentiable, strictly convex cost function, we proved in [1] that whenever the dynamics in (2) are such that

$$
k^{i}\left(-\omega_{i}^{*}\right)=\left[\left(c_{i}^{\prime}\right)^{-1}\left(-\omega_{i}^{*}\right)\right]_{s_{i}^{\min }}^{s_{\text {max }}}
$$

then the equilibrium values $s_{i}^{*}$ are optimal for the OSLC problem (4). Consequently, if the generation and load control schemes are designed such that the characteristic response at equilibrium satisfies (5), then these equilibria of (1)-(2) are guaranteed to be optimal solutions for (4).

Furthermore, if the solutions of (1)-(2) can be shown to converge to the equilibrium set, then we can also infer that they converge to optimality for the OSLC problem (4). This motivates our consideration in what follows of the stability properties of the set of equilibria of (1)-(2).

\section{STABILITy ANALYSiS}

In [1], we proved that local asymptotic stability, and thus from the analysis in Section III-C also convergence to optimality, can be guaranteed using the property of input-strict passivity. That is, we saw that if the supply dynamics (2) are input-strictly passive about an equilibrium point, then all solutions of (1)-(2) starting sufficiently close to the equilibrium are guaranteed to converge to the equilibrium set. We derived this convergence result by means of a Lyapunov stability analysis for the nonlinear system (1)-(2). However, we will now show that, whenever the system is operating in a regime for which a linearization is feasible, the passivity condition can be relaxed to less restrictive decentralized conditions. In particular, we will show that additional local information, accounting for the coupling strength between adjacent buses, allows one to derive decentralized stability certificates that guarantee convergence, which are consistently less conservative than passivity.

To see this, we require a smoothness assumption to enable linearizations to be taken.

Assumption 3: The functions $f^{i}$ and $g^{i}$ in (2) are continuously differentiable on open neighbourhoods of the equilibrium of (1)-(2) under consideration.

Remark 2: Note that Assumption 3 requires that the equilibrium under consideration be located at a point of continuous differentiability of the systems (2). In practice, this will often correspond to situations in which the steady state generation and load control values do not hit their saturation bounds. If this requirement is violated, then a nonlinear analysis, such as the Lyapunov approaches used in [1], will be needed ${ }^{2}$.

Under Assumption 3, the system (1)-(2) can be linearized about the equilibrium being considered. In order to study this linearization, let the notation $\tilde{q}=q-q^{*}$ denote the deviation of any quantity $q$ from its value $q^{*}$ at this equilibrium point.

We first introduce the quantities $H_{i}(s)$ to represent the transfer functions for the linearized versions of the systems in (2) from $-\tilde{\omega}_{i}$ to $\tilde{s}_{i}$. Note that $H_{i}(s)$ is also then trivially the

\footnotetext{
${ }^{2}$ It should be noted that analysis techniques on nonlinear and linearized models are of independent interest in power system analysis [14]. The former can allow larger disturbances but they can be difficult to apply or lead to conservative designs when more involved system models are used. The latter are valid for small disturbances but can lead to less conservative designs.
} 

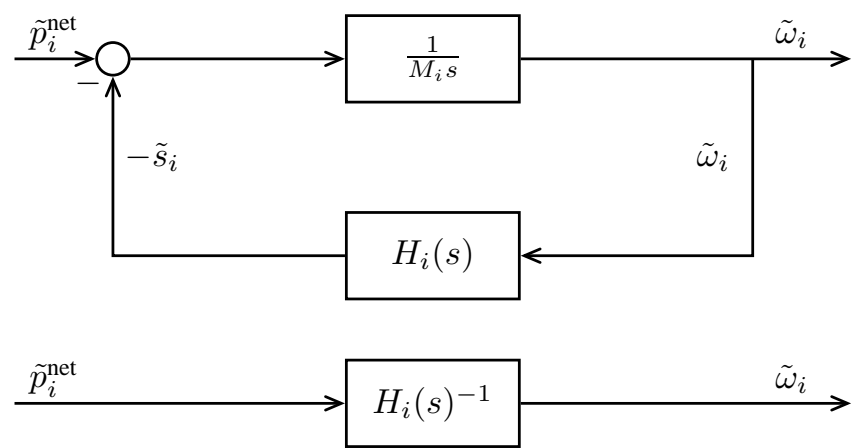

Fig. 1. Block diagrams of the dynamics $T_{i}(s)$ for $i \in \mathcal{G}$ (top figure) and $i \in \mathcal{L}$ (bottom figure). For simplicity of the illustration, we omit the dependence on the initial conditions $\tilde{\omega}_{i}(0)$ and $\tilde{x}^{i}(0)$.

transfer function from $\tilde{\omega}_{i}$ to $-\tilde{s}_{i}$. Since Assumption 3 ensures that such a linearization is possible, these $H_{i}(s)$ are known to be rational functions.

Let us then couple these supply dynamics with the frequency variation (1b) to form subsystems, represented by the transfer functions $T_{i}(s)$ from inputs given by the net power inflows, $\tilde{p}_{i}^{\text {net }}:=-\sum_{l: i \rightarrow l} \tilde{p}_{i l}+\sum_{l: l \rightarrow i} \tilde{p}_{l i}$, to outputs given by the local frequencies $\tilde{\omega}_{i}$. Due to the distinction between generation and load buses, it follows that this generates two distinct forms ${ }^{3}$ of transfer functions, which can be expressed as

$$
T_{i}(s)= \begin{cases}\frac{1 / s}{M_{i}+H_{i}(s) / s}, & i \in \mathcal{G} \\ H_{i}(s)^{-1}, & i \in \mathcal{L} .\end{cases}
$$

The corresponding block diagrams from which these expressions arise are illustrated in Fig. 1. Note that the condition in Assumption 2 is sufficient to ensure that the inverted transfer functions at the load buses in (6) are well-defined. Nonetheless, the subsystems represented by $T_{i}(s)$ for $i \in \mathcal{L}$ may in general still be non-causal (i.e. the transfer function $T_{i}(s)$ is not proper); this is not a problem within our analysis since the system described by (1)-(2) relating initial conditions to states is both well-defined and causal.

Remark 3: Exact analytical representations for the transfer functions $H_{i}(s)$ and $T_{i}(s)$ can be determined, in terms of the Jacobian matrices of the various functions appearing in (2), by applying the Laplace transform and rearranging the resulting linear equations. However, for simplicity of presentation, we suppress these details and instead work directly with the aggregate transfer functions (6).

Finally, consider the interconnection structure that couples the individual subsystems (6) through the linearized dynamics in (1a) and (1c) from frequency to negative power influxes. These interconnecting dynamics can be represented by the transfer function

$$
A \operatorname{diag}\left(\frac{B}{s}\right) A^{T}
$$

\footnotetext{
${ }^{3}$ Note that, as expected, the form of $T_{i}(s)$ for load buses is recovered from that for generator buses by letting the generation inertia $M_{i}$ tend to zero.
}

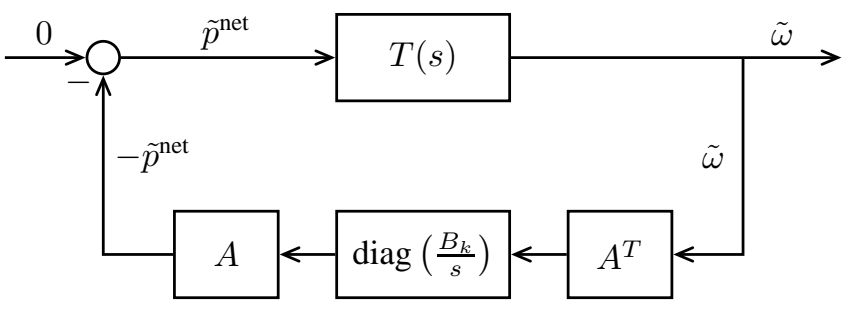

Fig. 2. Block diagram representing the linearized dynamics of the overall power system model (1)-(2). The initial conditions $\tilde{\eta}(0), \tilde{\omega}(0)$, and $\tilde{x}(0)$ are again omitted for simplicity of the illustration.

where $A$ is the incidence matrix of the network graph structure,

$$
A_{q r}= \begin{cases}+1, & \text { if } r=(q, \cdot) \in \mathcal{E} \\ -1, & \text { if } r=(\cdot, q) \in \mathcal{E} \\ 0, & \text { otherwise }\end{cases}
$$

and $B$ denotes the vector of susceptances $\left(B_{k}\right)_{k \in \mathcal{E}}$.

The overall power system model (1)-(2) is then represented by the negative feedback interconnection between the collection $T(s)=\operatorname{diag}\left(\left(T_{i}(s)\right)_{i \in N}\right)$ of subsystems (6) and the coupling dynamics (7). This feedback interconnection is illustrated in Fig. 2. We will formulate conditions on the frequency response of the transfer functions $T_{i}(s)$ under which stability of this interconnection is guaranteed. To enable us to do this, we introduce one final assumption, which defines the broad class of transfer functions that we consider.

Assumption 4: We assume that

- $T_{i}(s)$ are rational transfer functions with no poles in the closed right half-plane, for all $i \in \mathcal{N}$.

- $T_{i}(s)$ satisfies $^{4} T_{i}(0)>0$, for all $i \in \mathcal{N}$.

- $T_{i}(s)$ has relative degree ${ }^{5}$ at least -1 , for all $i \in \mathcal{L}$.

Remark 4: The first condition in Assumption 4 is closely linked to the open-loop stability that we assume for the systems in (2). The latter two conditions are technical properties that we require within our analysis. However, we shall see in Section $\mathrm{V}$ that these are general conditions which extend the input-strict passivity properties that we invoked for the stability analysis in [1]. Note in particular that the third condition is always satisfied for any load dynamics incorporating arbitrarily small damping, and hence can be expected to hold in most realistic scenarios.

Having specified the class of transfer functions that will be permitted, we are now ready to state our stability result, Theorem 1. This imposes decentralized conditions on the bus frequency responses $T_{i}(j \phi)$ by constraining those to lie in prescribed regions of the complex plane (denoted as $P_{\lambda}^{+}$in the theorem statement). In particular, these regions are halfplanes to the right of a line through the point -1 and are defined below (see also Fig. 3).

Definition 3: Let $\lambda_{\phi}$ be a line in the complex plane through the point -1 with non-zero gradient, i.e. $\lambda_{\phi}=\left\{-1+k v_{\phi}\right.$ : $k \in \mathbb{R}\}$ where $v_{\phi} \in \mathbb{C}$ is a constant with $\Im\left(v_{\phi}\right) \neq 0$. Then

\footnotetext{
${ }^{4}$ Note that any rational transfer function $T_{i}$ is always real when evaluated on the real axis, so there is no additional restriction here in assuming $T_{i}(0) \in \mathbb{R}$.

${ }^{5}$ The relative degree of a rational transfer function is defined as the polynomial degree of its denominator minus the polynomial degree of its numerator.
} 


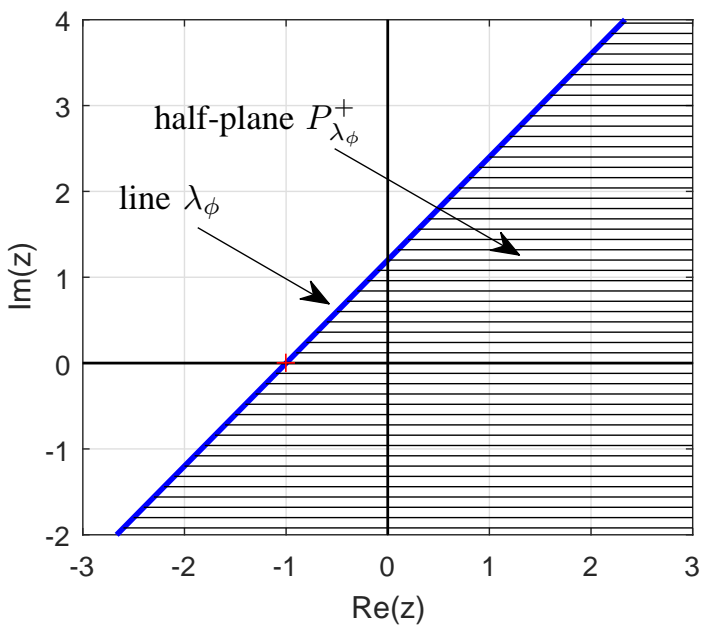

Fig. 3. The figure illustrates the region $P_{\lambda_{\phi}}^{+}$defined in Definition 3. In particular, $P_{\lambda_{\phi}}^{+}$is the half-plane to the right of line $\lambda_{\phi}$ through the point -1 .

$P_{\lambda_{\phi}}^{+}$denotes the open half-plane to the right of $\lambda_{\phi}$, i.e. $P_{\lambda_{\phi}}^{+}=$ $\left\{x \in \mathbb{C}: \Re(x)>\Re(y)\right.$ for some $\left.y \in \lambda_{\phi}\right\}$.

Theorem 1: Suppose that Assumptions 1-4 are all satisfied. Let $K_{i}:=2\left(\sum_{l: i \rightarrow l} B_{i l}+\sum_{l: l \rightarrow i} B_{l i}\right)$. Then the set of equilibrium points of the linearization of system (1)-(2) about $\left(\eta^{*}, \omega^{*}, x^{*}\right)$ with frequencies equal to $\omega^{*}$ is asymptotically stable if for each $\phi \in \overline{\mathbb{R}}_{+} \backslash\{0\}$ there exists a line $\lambda_{\phi}$ in $\mathbb{C}$ through the point -1 and with non zero gradient such that

$$
\frac{K_{i} T_{i}(j \phi)}{j \phi} \in P_{\lambda_{\phi}}^{+}
$$

for all $i \in \mathcal{N}$, where $P_{\lambda_{\phi}}^{+}$is the open half-plane to the right of $\lambda_{\phi}$ (defined in Definition 3).

Remark 5: As it will be discussed in detail in Section V-A, the condition in the theorem recovers and generalizes an input strict passivity condition on the load/generation dynamics $H_{i}(s)$. In particular, an input strict passivity condition would constrain the frequency response $H_{i}(j \phi)$ to lie in the right half-plane, whereas the fact that half-planes $P_{\lambda_{\phi}}^{+}$are allowed to have arbitrary orientation, and vary with frequency, reduces the conservatism in the analysis (examples will be given in Section V-B). This relaxation is due to the fact that the coupling gains between neighbouring buses, via the susceptances $B_{i j}$, are incorporated in the analysis.

Remark 6: Note that condition (8) provides a decentralized certificate for asymptotic stability. In particular, each bus can independently ensure that its frequency response satisfies (8) with respect to a particular collection of lines $\lambda_{\phi}$ (and corresponding half planes $P_{\lambda_{\phi}}^{+}$) that are specified a priori as part of the "grid code" that needs to be followed. The scaling factors $K_{i}=2\left(\sum_{l: i \rightarrow l} B_{i l}+\sum_{l: l \rightarrow i} B_{l i}\right)$ are calculated in terms of the susceptances of all lines entering or leaving the bus $i$, and so require knowledge of only the local network structure. Furthermore, observe that each bus is free to choose any dynamics so long as the frequency response satisfies (8). As such, the dynamics at the various buses are permitted to be heterogeneous.

Remark 7: Analogously to [15], the conditions (8) can give rise to an interpretation in terms of integral quadratic constraints. In turn, these can be verified by means of LMI (linear matrix inequalities) conditions via the generalized KYP Lemma [16]. Therefore, the stability conditions (8) can be efficiently checked either graphically or numerically (via the feasibility of a convex LMI problem).

If, furthermore, the global bus structure and all line susceptances are known to all buses priori, then Theorem 1 can be further relaxed to a less restrictive stability condition.

Theorem 2: Suppose that Assumptions 1-4 are all satisfied. Then the set of equilibrium points of the linearization of system (1)-(2) about $\left(\eta^{*}, \omega^{*}, x^{*}\right)$ with frequencies equal to $\omega^{*}$ is asymptotically stable if

$$
\frac{\mu^{\max } K_{i} T_{i}(j \phi)}{j \phi} \in P_{\lambda_{\phi}}^{+}
$$

holds for all $i \in \mathcal{N}$ and all $\phi \in \overline{\mathbb{R}}_{+} \backslash\{0\}$, where $K_{i}>0$ are constants, $\mu^{\max }=\rho\left(A \operatorname{diag}(B) A^{T} \operatorname{diag}\left(\frac{1}{K}\right)\right)$, and regions $P_{\lambda_{\phi}}^{+}$ are as defined in Theorem 1.

Remark 8: Note that if the constants $K_{i}$ are chosen as in Theorem 1, then the maximal eigenvalue $\mu^{\max }$ satisfies $\mu^{\max } \leq\left\|A \operatorname{diag}(B) A^{T} \operatorname{diag}\left(\frac{1}{K}\right)\right\|_{\infty} \leq 1$ so the stability condition (9) is generally less restrictive than (8). This makes clear the distinction between Theorems 1 and 2: Theorem 1 offers an entirely distributed stability certificate (8) whereby each bus need only know the susceptances of its neighboring lines, whereas Theorem 2 provides a less conservative stability certificate (9) provided that it is possible to share the description of the overall bus structure and line susceptances across the entire network (so as to be able to calculate $\left.\mu^{\max }\right)$. Analogously, it is also possible to view the comparison between Theorem 1 and the passivity analysis in [1] in this manner: input-strict passivity in [1] allowed stability to be guaranteed without any structural knowledge whatsoever, whereas Theorem 1 forms less restrictive stability criteria (8) whenever each bus knows its local line susceptances. In this way, we see that additional knowledge of the system structure can lead to less restrictive stability conditions.

Remark 9: It should be noted that in the case where the scaled bus dynamics $K_{i} T_{i}(s)$ are identical for some constants $K_{i}$, condition (9) becomes very similar to the necessary and sufficient condition for stability, thus illustrating a property of non-conservativeness. In particular, in this case it follows easily from the Nyquist stability criterion [17] that the necessary and sufficient condition for stability is that the LHS of (9) should not encircle the point -1 when evaluated on a Nyquist contour, with $\mu^{\max }$ any number in the spectrum of $\tilde{L}$, where $\tilde{L}=A \operatorname{diag}(B) A^{T} \operatorname{diag}\left(\frac{1}{K}\right)$. Condition (9) is equivalent to this condition but with $\mu^{\max }$ being any number in the interval $[0, \rho(\tilde{L})]$.

\section{DISCUSSION AND EXAMPLES}

\section{A. Connections of Section IV with passivity}

In this section we consider in detail how the stability conditions (8) and (9) compare to the assumption of inputstrict passivity that was imposed in the stability analysis in [1].

More precisely, suppose that the supply dynamics with transfer function $H_{i}(s)$ are input-strictly passive. We show below that this implies that Assumption 4 and condition (8) 
are satisfied, and then also discuss the extensions provided by Theorem 1.

We consider first Assumption 4. Input strict passivity implies $H_{i}(s)$ is strictly positive real [18], [19]. A simple consequence of this is that $H_{i}(0)>0$, whence we have from (6) that $T_{i}(0)>0$ for all $i \in \mathcal{N}$. Furthermore, for any rational transfer function $H_{i}(s)$, (6) defines a proper transfer function in the case $i \in \mathcal{G}$, which has relative degree greater than -1 . To see this also for the load buses, we note that any SISO positive real transfer function has relative degree equal to 0 or 1 [20], whence (6) will have relative degree of either 0 or -1 also for $i \in \mathcal{L}$. Finally, since $T_{i}(s)$ represents either an interconnection of an integrator and an input-strict passive system (for $i \in G$ ), or an inverse of a strictly positive real transfer function (for $i \in L$ ), one can deduce that $T_{i}(s)$ is positive real with no poles in the closed right half-plane. Therefore all conditions of Assumption 4 are satisfied.

We now verify that condition (8) is satisfied by investigating the possible values of the frequency responses $\frac{K_{i} T_{i}(j \phi)}{j \phi}$ when $H_{i}(s)$ is strictly positive real. The latter implies that $\Re\left(H_{i}(j \phi)\right)>0$ for all $\phi \in \mathbb{R}$. Considering the expressions (6), this implies, in both the generator and the load case, that the frequency response $T_{i}(j \phi)$ lies within the open right halfplane for all $\phi \in \mathbb{R}_{+} \backslash\{0\}$. It thus follows ${ }^{6}$ that, for all $i \in \mathcal{N}$,

$$
\left\{\frac{K_{i} T_{i}(j \phi)}{j \phi}: \phi \in \mathbb{R}_{+} \backslash\{0\}\right\} \subseteq\{z \in \mathbb{C}: \Im(z)<0\} .
$$

Note that the set on the right-hand side in (10) is the lower half-plane, which can be seen as the limit of the set $P_{\lambda}^{+}$in (8) as the slope of the line $\lambda$ tends to zero. Consequently, we see that the passivity condition assumed in [1] requires the frequency response in the left-hand side of (8) to be restricted to the half-plane below a horizontal line through -1 , whereas Theorem 1 allows this bounding line to be rotated arbitrarily about -1 and to vary with $\phi$. This therefore allows to deduce local asymptotic stability for more broad classes of dynamics.

Note, however, as discussed in Remark 2, that the results in [1] are of independent interest as the analysis is nonlinear, based on Lyapunov approaches, without requiring the existence of a linearized system.

\section{B. Numerical example illustrating the stability conditions}

Consider the second-order turbine-governor dynamics (e.g. $[13$, p. 382]) given by

$$
\begin{aligned}
& \dot{\alpha}_{i}=-\frac{1}{\tau_{g, i}} \alpha_{j}+\frac{1}{\tau_{g, i}} p_{i}^{c}, \\
& \dot{p}_{i}^{M}=-\frac{1}{\tau_{b, i}} p_{j}^{M}+\frac{1}{\tau_{b, i}} \alpha_{i},
\end{aligned}
$$

to represent power generation. Here $\alpha_{i}$ is the valve position of the turbine, the constants $\tau_{g, i}$ and $\tau_{b, i}$ represent lags in the dynamics of the governor and turbine respectively, and $p_{i}^{c}$ is a static function of frequency, corresponding to droop control. The uncontrollable frequency-dependent loads and generator damping are modeled by terms $d_{i}^{u}=D_{i} \omega_{i}$. For simplicity, we include no controllable loads, though analogous arguments

${ }^{6}$ From the positive realness and relative degree of $T_{i}(s)$ it follows that $\frac{K_{i} T_{i}(j \infty)}{j \infty} \geq 0$ so (8) is trivially satisfied at $\phi=\infty$.

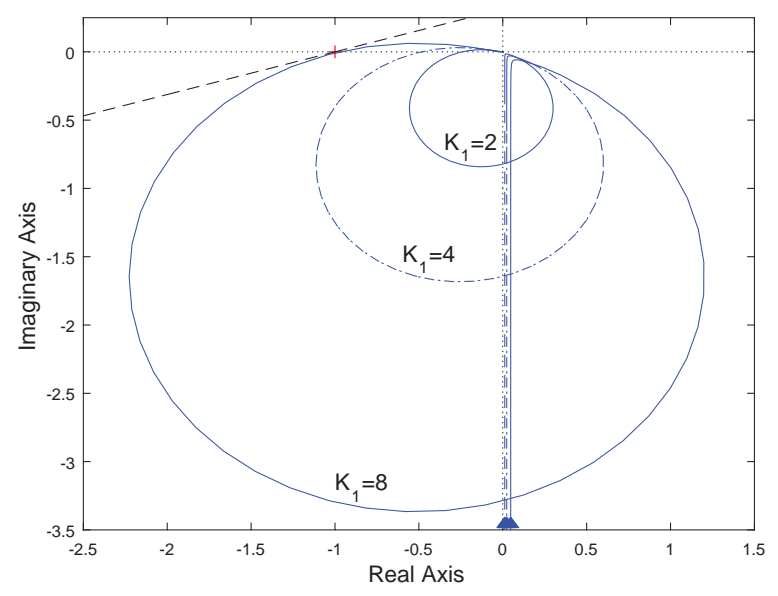

Fig. 4. The frequency response in (8) for a generation bus with varying values of $K_{i}$. Since the frequency response enters the upper half-plane, the supply dynamics are not passive. However, the frequency response lies to the right of the dashed line $\lambda_{\phi}$ for $K_{i} \leq 8$, indicating that stability can be verified for such dynamics using Theorem 1 .

could be followed were these present. Therefore the net supply is $s_{i}=p_{i}^{M}-d^{u}$. We then impose the constraint

$$
\left|p_{i}^{c}\left(\omega_{j}\right)-p_{i}^{c}\left(\omega_{i}^{*}\right)\right| \leq R_{i}\left|\omega_{i}-\omega_{i}^{*}\right|, i \in G
$$

on the generation command signals $p_{i}^{c}$. This condition was used in [21], where it was shown that $R_{i}<D_{i}$ for all $i \in$ $\mathcal{G}$ is sufficient to ensure convergence. Through the passivity approach in [1] we showed that this constraint can be relaxed, permitting the gain constants $R_{i}$ to take values larger than the damping coefficients $D_{i}$. In particular, the largest possible choice in order for the passivity argument to guarantee stability was seen to be

$$
R_{i}=\frac{\left(\tau_{g, i}+\tau_{b, i}\right)^{2}+2\left(\tau_{g, i}+\tau_{b, i}\right) \sqrt{\tau_{g, i} \tau_{b, i}}}{\tau_{g, i} \tau_{b, i}} D_{i} .
$$

We now provide an example that illustrates that, whenever a linearization of dynamics (1)-(2) is feasible, the permissible gain choices can be extended beyond those allowed by (13) using the stability result in Theorem 1.

For the example, choose the parameter values $\tau_{g, i}=\tau_{b, i}=$ $0.1, M_{i}=0.8$, and $D_{i}=2$ at a generation bus $i \in G$, and define the generation command signal to satisfy $\tilde{p}_{i}^{c}=-R_{i} \tilde{\omega}_{i}$ with $R_{i}=12 D_{i}$. That is, we assume equality in (12) and consider sufficiently large gain that (13) is violated (specifically, one-and-a-half times the maximum gain permitted by (13)), meaning that the passivity analysis cannot be used to guarantee stability. Taking the Laplace transform in (11) and applying (6) yields the transfer function

$$
T_{i}(s)=\frac{(0.1 s+1)(0.1 s+1)}{(0.1 s+1)(0.1 s+1)(0.8 s+2)+24} .
$$

The second and third points in Assumption 4 are obvious, while to verify the first requirement, note that the poles of (14) are exactly the roots of the cubic polynomial $(0.1 s+1)(0.1 s+$ $1)(0.8 s+2)+24=0$. Solving this equation gives the poles of $(14)$ as $-22.31,-0.09650 \pm 12.07 j$, all of which lie in the open left half-plane. Thus Assumption 4 holds for (14). Now, 
let us define the line ${ }^{7}$

$$
\lambda_{\phi}:=\{z \in \mathbb{C}: \Im(z)=0.3125(\Re(z)+1)\}
$$

for all $\phi \in \overline{\mathbb{R}}_{+} \backslash\{0\}$. This line is indicated in Fig. 4, along with the frequency response appearing in (8) for varying values of $K_{i}$. As noted, passivity is violated in this case and so these frequency responses leave the lower half-plane. Nonetheless, the frequency response lies wholly in the halfplane $P_{\lambda_{\phi}}$ to the right of $\lambda_{\phi}$ for all $K_{i} \leq 8$. For this bus, the stability certificate (8) is thus verified provided that its adjacent line susceptances satisfy $K_{i} \leq 8$. Therefore, it follows from Theorem 1 that if similarly all other buses satisfy condition (8) with the line $\lambda_{\phi}$ in (15) then the overall interconnected system will be asymptotically stable.

The example thus illustrates that decentralized stability conditions with reduced conservatism can be derived by taking into account additional local information, such as the coupling gain between buses via the line susceptances.

\section{Application to the NPCC 48 machine system}

To further demonstrate the relevance of our analysis, we apply the decentralized stability conditions derived in the paper to a realistic power system model, showing how these conditions can lead to a scalable design with a plug and play capability. In particular, we consider the NPCC (Northeast Power Coordinating Council) 48 machine system with realistic data provided by the power system toolbox ${ }^{8}$ [22]. This network has 22 buses with turbine governor systems described by fifth order models. The transfer functions $H_{i}(s)$ relating $-\tilde{\omega}_{i}$ to the supply $\tilde{s}_{i}$ can be deduced from the prameter values provided for each of these buses and it can be verified that these dynamics are passive for 20 out of the 22 buses and non-passive at 2 buses (the Nyquist plots of $H_{i}(s)$ at the two buses with non-passive ${ }^{9}$ dynamics are shown in Fig. 5).

In order to apply the stability condition in Theorem 1, the transfer functions $\frac{K_{i} T_{i}(s)}{s}$, where $T_{i}(s)$ is given in (6) and $K_{i}$ in the Theorem statement, were obtained for all 22 buses and the corresponding Nyquist plots were considered. It was found that the stability condition in Theorem 1 was satisfied with an increase by $18 \%$ in the damping of one of the non passive buses. This is illustrated in Fig. 6, where it can be seen that the Nyquist plots for $\frac{K_{i} T_{i}(s)}{s}$ for all the 22 buses lie in the half-plane to the right of a line with gradient 0.3 crossing the real axis at -1 .

It should be noted that the significance of this line is that it provides a decentralized stability condition for the network that also leads to a scalable design with a plug and play capability. In particular, if part of the network is modified or expanded stability will still be guaranteed if we ensure that at each bus the locally defined transfer function $\frac{K_{i} T_{i}(s)}{s}$ has a Nyquist plot (for positive frequencies) to the right of this line. That is, we only need to satisfy a decentralized condition and hence only need to adjust the control mechanism for the part of the network that has been modified.

\footnotetext{
${ }^{7}$ The form of the dynamics here means that in this case a single line $\lambda_{\phi}$ for all values of $\phi$ will suffice. In general, however, broader classes of dynamics can be permitted by selecting different lines $\lambda_{\phi}$ for different ranges of $\phi$.

${ }^{8}$ The data for the network can be found in the toolbox file datanp48.

${ }^{9}$ See also [1] for the Nyquist plots of $H_{i}(s)$ at buses with passive dynamics.
}

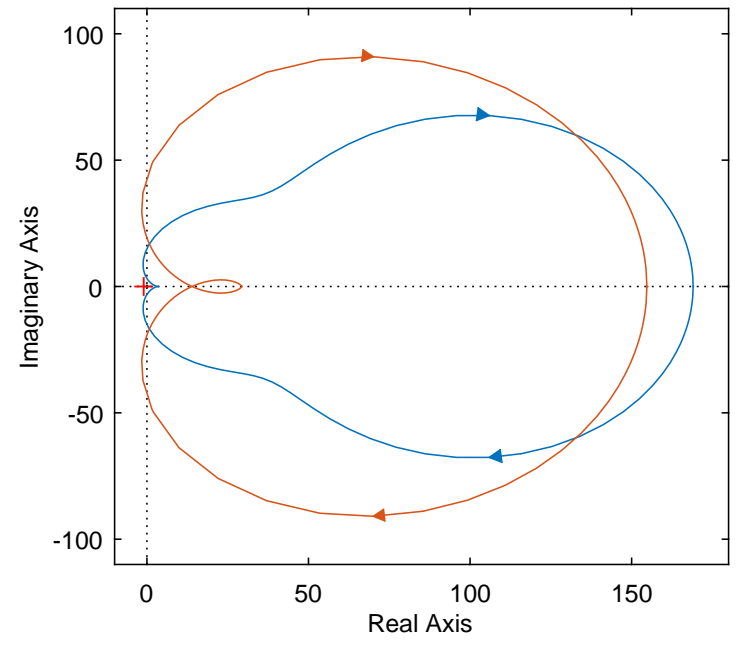

Fig. 5. Nyquist diagram of the net supply dynamics $H_{i}(s)$ for buses 26 and 27 respectively in the NPCC network. It can be seen that these dynamics are non-passive.

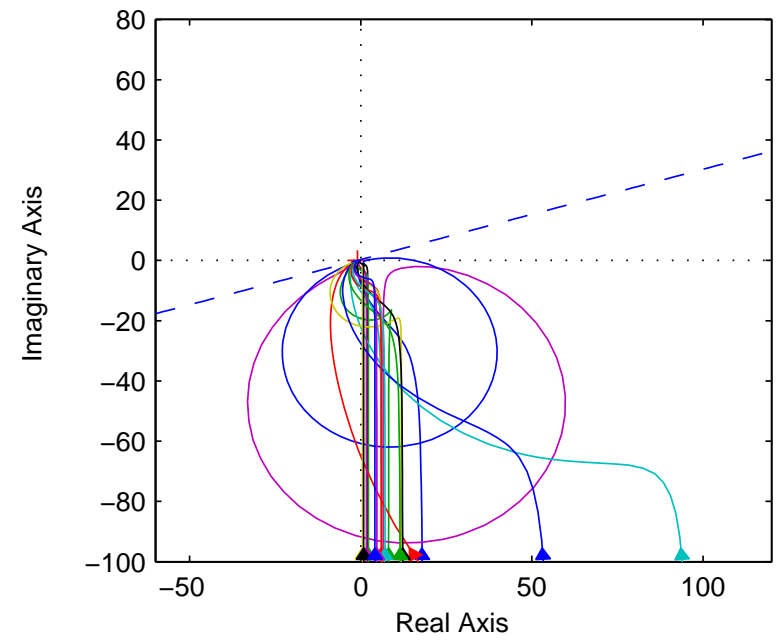

Fig. 6. Nyquist diagram (positive frequencies) of $\frac{K_{i} T_{i}(s)}{s}$ for the 22 buses with turbine governor systems in the NPCC network. It can be seen that the plots lie in the half-plane to the right of a line crossing the real axis at -1 .

We now compare the analysis above with the one based on passivity arguments. In our companion paper [1], it was discussed that if we provide stability guarantees by requiring all the bus dynamics to be passive, the damping coefficients for the two buses with non-passive dynamics need to be increased by $37 \%$ and $28 \%$ respectively. Theorem 1 provides distributed stability guarantees without any increase in damping for the first bus and with only $18 \%$ increase for the second. This shows how the approach in this paper can provide less conservative small signal decentralized stability conditions relative to the passivity conditions in [1].

\section{CONCLUSIONS}

We have considered the problem of designing distributed generation and demand control schemes for primary frequency 
regulation in power networks, such that asymptotic stability is guaranteed while ensuring optimality of power allocation. We have derived decentralized conditions under which equilibrium points of the overall system are guaranteed to be asymptotically stable. The stability analysis was conducted using frequency response techniques applied to a linearized form of the system dynamics. In particular, we have shown that, when such a linearization is feasible, additional local information can be exploited to derive decentralized stability conditions that are less restrictive than a passivity condition on the bus dynamics. This therefore allows to deduce convergence in a general network, by means of local feedback rules, for broader classes of generation and demand dynamics, thus reducing the conservatism in the analysis and design.

\section{APPENDIX: PROOFS OF OUR MAIN RESULTS}

In the appendix we provide detailed proofs of our main results, Theorems 1 and 2 .

Proof of Theorem 1: The proof makes use of ideas analogous to those in [15] and references therein, however the presence of multiple equilibrium points, non proper transfer functions in $T_{i}(s)$, and integrator dynamics introduce additional complications in the analysis that need to be explicitly addressed. In particular, we show that the closed loop transfer function of the linearized system relating the initial conditions with the frequency $\omega$ has no poles in the closed right halfplane. This is then used to deduce asymptotic stability of the set of equilibrium points considered in the theorem.

We start by considering a linearization of system (1)(2) about the equilibrium point used in Assumption 1. By taking Laplace transforms it can be shown that the frequency deviation $\tilde{\omega}$ can be written in terms of the initial conditions $\tilde{\eta}(0), \tilde{\omega}(0)$, and $\tilde{x}(0)$ as

$$
\begin{aligned}
\hat{\tilde{\omega}}=F(s)\left[A \operatorname{diag}\left(\frac{B}{s}\right)\right. & \hat{\eta}(0)+\operatorname{diag}\left(\left(M_{i}\right)_{i \in \mathcal{N}}\right) \hat{\omega}(0) \\
& \left.+\operatorname{diag}\left(\left(m_{i} H_{i}(s)+n_{i}\right)_{i \in \mathcal{N}}\right) \hat{x}(0)\right],
\end{aligned}
$$

in terms of constants ${ }^{10} m_{i}, n_{i}$ and the function

$$
F(s)=\left(I+T(s) A \operatorname{diag}\left(\frac{B}{s}\right) A^{T}\right)^{-1} T(s) .
$$

Observe also that the asymptotic stability of the dynamics (2) implies that the corresponding transfer functions in the linearization, $H_{i}(s)$, have no poles in the closed right half-plane.

The proof of the theorem has three parts. We show in Step 1 that $F(s)$ and (16) have no poles at 0 , and we show in Step 2 that $F(s)$ has no poles in the closed right half-plane. These results imply $\tilde{\omega} \rightarrow 0$ and we use this in Step 3 to deduce convergence to the set of equilibrium points:

Step 1: We show that $F(s)$ and (16) have no poles at $s=0$. Note that $\frac{1}{s} F(s)=\left(s I+T(s) A \operatorname{diag}(B) A^{T}\right)^{-1} T(s)$. Since the underlying graph is connected, $\operatorname{Adiag}(B) A^{T}$ has a simple eigenvalue at the origin. Using also the fact that $T(0)>0$

\footnotetext{
${ }^{10}$ The explicit values of these constants are $m_{i}=\frac{\partial f_{i}}{\partial(-\omega)}\left(x^{i, *},-\omega_{i}^{*}\right)^{-1}$ and $n_{i}=-\frac{\partial f_{i}}{\partial(-\omega)}\left(x^{i, *},-\omega_{i}^{*}\right)^{-1} \frac{\partial g_{i}}{\partial(-\omega)}\left(x^{i, *},-\omega_{i}^{*}\right)$, but this precise form is irrelevant for the argument that follows.
}

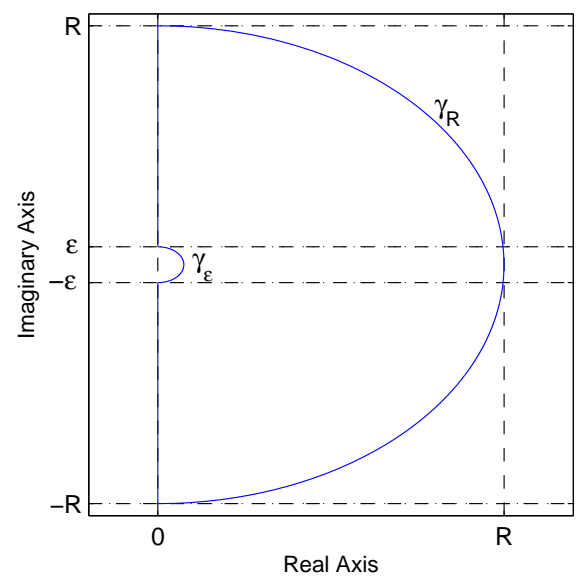

Fig. 7. The Nyquist contour $\Gamma_{\epsilon, R}$, which is composed of the small arc $\gamma_{\epsilon}$, the large arc $\gamma_{R}$, and the imaginary axis intervals $[-j R,-j \epsilon],[j \epsilon, j R]$.

we have that $\frac{1}{s} F(s)$ has a simple pole at $s=0$, hence $F(s)$ has no poles at $s=0$. In order to show that the expression in (16) also has no poles at $s=0$ we need to show that $G(s):=F(s) A \operatorname{diag}(B / s)$ has no poles at $s=0$. This can be seen by making use of the fact that for a matrix $M \in$ $\mathbb{C}^{n \times m}$ the limit $\lim _{s \rightarrow 0}\left(s I+M^{*} M\right)^{-1} M^{*}$ always exists [23] (this is equal to the pseudo inverse of $M$ ). Hence noting that $\left.\lim _{s \rightarrow 0} G(s)=\lim _{s \rightarrow 0} \bar{T}\left(s I+\bar{A}^{*} \bar{A}\right)^{-1} \bar{A}^{*} \bar{B}\right)$, where $\bar{T}:=$ $\sqrt{T(0)}, \bar{B}:=\operatorname{diag}(\sqrt{B}), \bar{A}^{*}:=\bar{T} A \bar{B}$ we see that $G(s)$ exists at $s=0$ and hence $G(s)$ has no poles at that point. Therefore since $H_{i}(s)$ also has no poles at $s=0$ the expression in (16) has no poles at the origin.

Step 2: We use a Nyquist argument to show that $F(s)$ has no poles in the closed right half-plane. Since we already showed in Step 1 that $F(s)$ has no poles at $s=0$, it is sufficient to show that $F(s)$ has no nonzero poles in the closed right half-plane. We denote $\Gamma_{\epsilon, R}$ the usual Nyquist contour indented in the right half-plane about the pole at $s=0$, as illustrated in Fig. 7. Then the multivariable Nyquist criterion [17] guarantees that (17) has no nonzero poles in the closed right half-plane if the point -1 is not encircled by the eigenloci of the return ratio

$$
L(s)=T(s) A \operatorname{diag}\left(\frac{B}{s}\right) A^{T}
$$

evaluated along $\Gamma_{\epsilon, R}$ with $\epsilon \rightarrow 0$ and $R \rightarrow \infty$. It is convenient to write the return ratio in the form $L(s)=\frac{T(s)}{s} A \operatorname{diag}(B) A^{T}$ and to note that

$$
\left[A \operatorname{diag}(B) A^{T}\right]_{q r}= \begin{cases}\sum_{l: q \rightarrow l} B_{q l}+\sum_{l: l \rightarrow q} B_{l q}, & r=q \\ -B_{q r}, & r \neq q\end{cases}
$$

which is a diagonally dominant matrix with nonnegative diagonal, and hence it is positive semidefinite.

Let us consider first the contribution of the two arcs $\gamma_{\epsilon}$ and $\gamma_{R}$ to the eigenloci. Firstly, since the transfer functions $T_{i}(s)$ have relative degree at least -1 , they satisfy $\frac{T_{i}(s)}{s} \rightarrow$ constant and hence $L(s) \rightarrow$ constant and $\sigma(L(s)) \rightarrow$ constant as $|s| \rightarrow \infty$. Therefore, in the limit as $R \rightarrow \infty$, the eigenloci evaluated along the large arc $\gamma_{R}$ become just constant points. Secondly, we note that $L(s)$ has only a simple pole at $s=0$. 
Therefore, the eigenloci travel along an arc by $\pi$ radians as $s$ traverses the small arc $\gamma_{\epsilon}$ in the limit as $\epsilon \rightarrow 0$. Moreover, we know that each $T_{i}(0)>0$, whence by continuity there exists sufficiently small $\delta>0$ such that $\frac{T_{i}(\epsilon)}{\epsilon}>0$ for all $\epsilon \in(0, \delta)$. Thus, since we already know $A \operatorname{diag}(B) A^{T}$ to be positive semidefinite, it follows that $\sigma(L(\epsilon)) \geq 0$ for all $\epsilon \epsilon$ $(0, \delta)$. This guarantees that, in the limit as $\epsilon \rightarrow 0$, the arc corresponding to the eigenloci along $\gamma_{\epsilon}$ is closed through the right half-plane, which lies to the right of the point -1 .

From these arguments, it follows that the closed-loop transfer function (17) has no nonzero poles in the right half-plane if the intersection $^{11}[-\infty,-1] \cap\left\{\sigma(L(j \phi)): \phi \in \overline{\mathbb{R}}_{+} \backslash\{0\}\right\}$ is empty. This will hold in particular if

$$
-1 \notin \operatorname{Co}(\sigma(L(j \phi)) \cup\{0\}) \text { for all } \phi \in \overline{\mathbb{R}}_{+} \backslash\{0\}
$$

since the set on the right-hand side in (20) is convex and includes the point 0 but does not include -1 .

We now develop a condition under which (20) will hold by bounding the spectrum $\sigma(L(j \phi))$ through use of ideas from matrix analysis [12]. To do this, we observe that, by making a similarity transformation with the matrix $\operatorname{diag}\left(\frac{1}{\sqrt{K}}\right)=$ $\operatorname{diag}\left(\left(\frac{1}{\sqrt{K_{i}}}\right)_{i \in \mathcal{N}}\right)$, the spectrum of $L(s)$ is the same as that of

$$
L^{\prime}(s)=\operatorname{diag}\left(\frac{\left(K_{i} T_{i}(s)\right)_{i \in \mathcal{N}}}{s}\right) \Xi,
$$

where $\Xi=\operatorname{diag}\left(\frac{1}{\sqrt{K}}\right) A \operatorname{diag}(B) A^{T} \operatorname{diag}\left(\frac{1}{\sqrt{K}}\right)$.

Let us now consider $\Xi$, which is a real symmetric matrix. Since $\Xi$ is normal its numerical range can be determined as $W(\Xi)=\operatorname{Co}(\sigma(\Xi))$. Additionally, recalling that $A \operatorname{diag}(B) A^{T} \succeq 0$ and noting that $\operatorname{diag}\left(\frac{1}{\sqrt{K}}\right)$ is diagonal with positive diagonal, it follows that $\Xi$ is also positive semidefinite. Finally, the spectral radius of $\Xi$ can be bounded as

$$
\begin{aligned}
\rho(\Xi) & =\rho\left(\operatorname{diag}\left(\frac{1}{K}\right) A \operatorname{diag}(B) A^{T}\right) \\
& \leq\left\|\operatorname{diag}\left(\frac{1}{K}\right) A \operatorname{diag}(B) A^{T}\right\|_{\infty} \\
& =\max _{i} \frac{2}{K_{i}}\left(\sum_{l: i \rightarrow l} B_{i l}+\sum_{l: l \rightarrow i} B_{l i}\right)=1,
\end{aligned}
$$

invoking in the inequality the induced $\infty$-norm bound for the spectral radius. Therefore, $\Xi$ is positive semidefinite with $\rho(\Xi) \leq 1$, which implies that $\sigma(\Xi) \subseteq[0,1]$. Consequently, we see that $\Xi$ has numerical range

$$
W(\Xi) \subseteq[0,1]
$$

Next, we note that the spectrum of a product of a diagonal and a positive semidefinite matrix is included in the product of

\footnotetext{
${ }^{11}$ Note that here we exploit the symmetry of the spectrum of $L(j \omega)$ about the real axis to reduce the condition (20) to require only evaluation of the spectrum over $\overline{\mathbb{R}}_{+} \backslash\{0\}$. The fact that $\infty$ is included within the set $\overline{\mathbb{R}}_{+} \backslash\{0\}$ enables condition (20) to guarantee that the constant points arising from $\chi$, as discussed above, also do not lie within the interval $[-\infty,-1]$.
}

the respective numerical ranges [12, Corollary 1.7.7]. Hence

$$
\begin{aligned}
\sigma\left(L^{\prime}(j \phi)\right) & \subseteq W\left(\operatorname{diag}\left(\frac{\left(K_{i} T_{i}(j \phi)\right)_{i \in \mathcal{N}}}{j \phi}\right)\right) W(\Xi) \\
& \subseteq \operatorname{Co}\left(\left\{\frac{K_{i} T_{i}(j \phi)}{j \phi}: i \in \mathcal{N}\right\} \cup\{0\}\right)
\end{aligned}
$$

using (23) and the fact that for a diagonal matrix its numerical range is equal to the convex hull of its elements (follows easily from the definition of the numerical range).

Therefore, condition (20) holds if

$$
-1 \notin \operatorname{Co}\left(\left\{\frac{K_{i} T_{i}(j \phi)}{j \phi}: i \in \mathcal{N}\right\} \cup\{0\}\right)
$$

for all $\phi \in \overline{\mathbb{R}}_{+} \backslash\{0\}$. It is clear that (25) will be satisfied if $\frac{K_{i} T_{i}(j \phi)}{j \phi}$ lie for all $i$ to the right of a line of nonzero gradient passing through -1 , which we denote by $\lambda_{\phi}$. This is condition (8).

Step 3: We therefore deduce from Step 1, Step 2 and the stability of $H_{i}(s)$ that (16) has no poles in the closed right half-plane. Hence, for all initial conditions, the frequency deviations $\tilde{\omega}$ tend to zero as $t \rightarrow \infty$ whenever (8) is satisfied. Furthermore, since asymptotically stable linear systems obey the converging input-converging state property [24], then $\tilde{x}$ and $\tilde{s}$ converge to zero also. Additionally, since for a linear system the convergence $\tilde{\omega} \rightarrow 0$ is always exponential, it follows from (1a) that $\tilde{\eta}$ and $\tilde{p}$ must tend to some constant values. Therefore, for the linearized system we deduce that the equilibrium set is asymptotically stable whenever the stability certificate $\frac{K_{i} T_{i}(j \phi)}{j \phi} \in P_{\lambda_{\phi}}^{+}$given in (8) is satisfied by all buses $i \in N$ for all $\phi \in \overline{\mathbb{R}}_{+} \backslash\{0\}$.

Remark 10: It should be noted that the proof does not make use of the fact that $\eta_{i j}=\theta_{i}-\theta_{j}$. As discussed in Part I, Remark 12, it can be shown that by making use of this property the equilibrium value $\eta^{*}$ is unique for a given equilibrium frequency $\omega^{*}$.

Proof of Theorem 2: The proof of this result follows from that of Theorem 1 but with (22) replaced by the exact expression $\rho(\Xi)=\mu^{\max }$ and noting that $K_{i}$ can now be any positive constant. Then (23) is replaced by $W(\Xi) \subseteq\left[0, \mu^{\max }\right]$, whence the required factor of $\mu^{\max }$ is inherited in (24) and (25). The remainder of the proof is as before.

\section{REFERENCES}

[1] A. Kasis, E. Devane, C. Spanias, and I. Lestas, "Primary frequency regulation with load-side participation Part I: stability and optimality" IEEE Transactions on Power Systems, 2016 (in press). An extended version is available online at http://arxiv.org/abs/1602.02800 .

[2] H. Miyagi and A. R. Bergen, "Stability studies of multimachine power systems with the effects of automatic voltage regulators," IEEE Transactions on Automatic Control, vol. 31, no. 3, pp. 210-215, 1986.

[3] D. J. Hill and C. N. Chong, "Lyapunov functions of Lur'e-Postnikov form for structure preserving models of power systems," Automatica, vol. 25, no. 3, pp. 453-460, May. 1989

[4] A. J. van der Schaft and B. Maschke, "Port-Hamiltonian systems on graphs," SIAM Journal on Control and Optimization, vol. 51, no. 2, pp. 906-937, 2013.

[5] B. Maschke, R. Ortega, and A. J. van der Schaft, "Energy-based Lyapunov functions for forced Hamiltonian systems with dissipation," IEEE Transactions on Automatic Control, vol. 45, no. 8, pp. 1498-1502, 2000.

[6] Y. Wang, D. Cheng, C. Li, and Y. Ge, "Dissipative Hamiltonian realization and energy-based L2-disturbance attenuation control of multimachine power systems,' IEEE Transactions on Automatic Control, vol. 48, no. 8, pp. $1428-1433,2003$. 
[7] S. Fiaz, D. Zonetti, R. Ortega, J. M. A. Scherpen, and A. J. van der Schaft, "A port-Hamiltonian approach to power network modeling and analysis," European Journal of Control, vol. 19, no. 6, pp. 477-485, 2013.

[8] S. Y. Caliskan and P. Tabuada, "Compositional transient stability analysis of multimachine power networks," IEEE Transactions on Control of Network Systems, vol. 1, no. 1, pp. 4-14, 2014.

[9] J. Liu, B. H. Krogh, and B. E. Ydstie, "Decentralized robust frequency control for power systems subject to wind power variability," Proceedings of the IEEE Power and Energy Society General Meeting (PES), pp. 1-8, 2011.

[10] M. Andreasson, R. Wiget, D. V. Dimarogonas, K. H. Johansson, and G. Andersson, "Distributed primary frequency control through multiterminal HVDC transmission systems," Proceedings of the American Control Conference (ACC), pp. 5029-5034, 2015.

[11] P. Kundur, Power System Stability and Control. McGraw-Hill New York, 1994.

[12] R. A. Horn and C. R. Johnson. Topics in Matrix Analysis, Cambridge University Press, 1991.

[13] A. R. Bergen and V. Vittal, Power Systems Analysis. Prentice Hall, 1999.

[14] P. Kundur et al., "Definition and Classification of Power system Stability," IEEE Transactions on Power Systems, vol. 19, no. 3, pp. 1387-1401, Aug. 2004.

[15] I. Lestas, "Large scale heterogeneous networks, the Davis-Wielandt shell, and graph separation," SIAM Journal on Control and Optimization, vol. 50, no. 4, pp. 1753-1774, Jul. 2012.

[16] T. Iwasaki and S. Hara, "Generalized KYP Lemma: unified frequency domain inequalities with design applications," IEEE Transactions on Automatic Control, vol. 50, no. 1, pp. 41-59, Jan. 2005.

[17] C. A. Desoer and Y. T. Wang, "On the generalized Nyquist stability criterion,", IEEE Transactions on Automatic Control, vol. AC-25, no. 2, pp. 187-196, Apr. 1980

[18] N. Kottenstette and P. J. Antsaklis, "Relationships between positive realm passive, dissipative, and positive systems", Proceedings of the American Control Conference, pp. 409-416, Jul. 2010.

[19] C. A. Desoer and M. Vidyasagar, Feedback Systems: Input-Output Properties. Elsevier, 1975.

[20] H. K. Khalil, Nonlinear Systems. Prentice Hall, 1996

[21] C. Zhao, and S. Low, "Optimal decentralized primary frequency control in power networks," Proceedings of the 53rd IEEE Conference on Decision and Control, pp 2467-2473, Dec. 2014.

[22] K. W. Cheung, J. Chow, and G. Rogers, Power System Toolbox, v. 3.0 Cherry Tree Scientific Software, 2009.

[23] S. L. Campbell and C. D Meyer, Generalized Inverses of Linear Transformations. SIAM, Classics in Applied Mathematics, 2009.

[24] E. D. Sontag, "State-space and I/O stability for nonlinear systems," Feedback Control, Nonlinear Systems, and Complexity, vol. 202, Jan. 1995. 